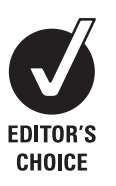

- A supplemental figure is published online only at http:// bjo.bmj.com/content/vol93/ issue6

Department of Ophthalmology, Kyoto University Graduate School of Medicine, Kyoto, Japan

Correspondence to: Dr K Nishijima, Department of Ophthalmology, Kyoto University Graduate School of Medicine, 54 Shogoin-Kawahara-cho, Sakyoku, Kyoto, 606-8507, Japan; nissi@kuhp.kyoto-u.ac.jp

Accepted 20 January 2009 Published Online First 12 February 2009

\title{
Randomised controlled trial of posterior sub-Tenon triamcinolone as adjunct to panretinal photocoagulation for treatment of diabetic retinopathy
}

\author{
N Unoki, K Nishijima, M Kita, K Suzuma, D Watanabe, H Oh, T Kimura, A Sakamoto, \\ $\mathrm{N}$ Yoshimura
}

\begin{abstract}
Aims: To evaluate the efficacy of a single posterior subTenon capsule injection of triamcinolone acetonide (PSTA) before panretinal photocoagulation (PRP).

Methods: This 6-month study involved the randomisation of 82 eyes of 41 patients, with bilateral severe nonproliferative diabetic retinopathy or proliferative diabetic retinopathy to a single PSTA $20 \mathrm{mg}$ or to no injection before PRP. The primary end-point was change in bestcorrected visual acuity (BCVA) at 6 months compared with that at baseline using the logarithm of the minimum angle of resolution (logMAR). Secondary end-points were changes in retinal thickness and intraocular pressure.

Results: The mean changes in logMAR BCVA at 6 months compared with that at baseline were a worsening of 0.010 (SD 0.029) in the control group (no injection) and an improvement of $0.072(0.028)$ in the PSTA group $(p=0.04)$. The mean changes in foveal thickness at 6 months compared with baseline measurements were an increase of 32.8 (82.8) $\mu \mathrm{m}$ in the control group and a lessening of 9.7 (85.6) $\mu \mathrm{m}$ in the PSTA group ( $p=0.03)$.
\end{abstract}

Conclusions: PSTA before PRP appears to be beneficial in preventing PRP-induced visual loss in eyes with diabetic retinopathy by reducing the chance of macular thickening.

Diabetic retinopathy is still a leading cause of blindness, even though the Early Treatment Diabetic Retinopathy Study (ETDRS) demonstrated that scatter laser panretinal photocoagulation (PRP) reduced the risk of severe visual loss in patients with high-risk proliferative diabetic retinopathy. ${ }^{1-3}$ Although PRP may prevent or halt proliferation in affected retinas, it sometimes causes or aggravates macular oedema, which is the main cause of acute visual disturbance. ${ }^{4-7}$

A number of studies suggested that postlaser release of inflammatory factors, an accumulation of leucocytes in the non-photocoagulated posterior pole, and upregulation of angiogenic growth factors, such as vascular endothelial growth factor, play an important role in the pathogenesis of the oedema, although the exact mechanism following PRP has not been elucidated. ${ }^{8-12}$

Wilson and colleagues ${ }^{13}$ reported that an intravitreal steroid in an animal model reduced bloodretinal barrier breakdown that was induced by retinal photocoagulation. Moreover, others reported the efficacy of periocular injection of triamcinolone acetonide as an adjunct to PRP for treatment of diabetic retinopathy. ${ }^{14-16}$ However, studies on the efficacy and safety of periocular injection of triamcinolone acetonide as adjunctive treatment to PRP are insufficient. This prospective randomised controlled trial was designed to evaluate the effectiveness of posterior sub-Tenon injection of triamcinolone (PSTA) against PRPinduced visual loss and macular thickening in patients with severe non-proliferative or proliferative diabetic retinopathy

\section{METHODS}

\section{Study design}

This trial was a randomised, contralateral eye controlled, open-label, parallel group study. Patients were recruited from the Kyoto University Hospital between July 2006 and October 2007. The trial was conducted in conformance with the tenets of the Declaration of Helsinki and the Japanese guideline. Approval was obtained from the Ethics Committee at Kyoto University, and each patient provided signed informed consent before study entry. This trial is registered at UMIN Clinical Trial Registry (C000000455, http://www.umin.ac. jp/ctr/index-j.htm).

\section{Subject selection}

Patients 20 years or older of either gender with type 1 or type 2 diabetes were eligible. All patients in the study underwent a complete ophthalmic examination, including best-corrected visual acuity (BCVA), slit-lamp biomicroscopy, funduscopy, applanation tonometry, fluorescein angiography and ocular coherence tomography (OCT) before recruitment; they had to have severe non-proliferative diabetic retinopathy (NPDR) or proliferative diabetic retinopathy (PDR) with clear ocular media and no other disease in either eye. The patients could have clinically significant macular oedema (CSMO) as defined by the ETDRS only if it was present in both eyes. Principal exclusion criteria included a history of panretinal or focal photocoagulation; a history of vitrectomy; presence of vitreous haemorrhage; signs of vitreomacular traction; periocular or intraocular steroid within the past 6 months; poorly controlled diabetes (defined as a haemoglobin $A_{1 c}$ level $>10 \%$ ); a history of glaucoma or ocular hypertension; and BCVA that differed between eyes by more than two Snellen lines. 


\section{Randomisation and masking}

Eyes were allocated to one of two groups (PSTA or no injection) by a stratification method according to BCVA converted into the logarithm of the minimum angle of resolution (logMAR) and foveal thickness on OCT at baseline. Under this system, the fellow eyes were inevitably allocated to the other group. Neither subjects nor investigators were masked, but those who tested visual acuity, OCT technicians and statistical analysers were masked as to treatment assignment of the eyes.

\section{Study treatment}

For posterior sub-Tenon capsule injection under topical anaesthesia, $20 \mathrm{mg}$ of triamcinolone acetonide (TA) (Kenacort: Bristol-Myers Squibb, New York) in a volume of $0.5 \mathrm{ml}$ was injected in the inferotemporal quadrant; this was done 1 week before the first PRP session. Eyes in the control group received no injections.

\section{Non-study treatments}

The PRP was performed four times at 2-week intervals in both eyes. The spot size on the retina was $200-300 \mu \mathrm{m}$, the power of the laser was 150-200 $\mathrm{mW}$, and the duration of the application was $0.2 \mathrm{~s}$ with the fundus laser lens (Super Quad 160: Volk, Mentor, Ohio) and the argon laser (Novus Omni: Coherent, Santa Clara, California) mounted on a slit lamp. The number of spots in each session was approximately 400, so the total number of burns after completion of the four sessions was approximately 1600. Topical anaesthesia was used in all cases, and all patients were treated as outpatients. If CSMO were present in both eyes at baseline, focal or grid laser therapy was performed at the first session of PRP.

\section{Outcome measurements and follow-up}

The primary end-point of the trial was a change in logMAR BCVA at 6 months' follow-up, compared with that at baseline. BCVA was assessed by Snellen visual acuity chart and was converted into $\log M A R$. The secondary end-points were retinal (foveal, parafoveal, perifoveal) thickness measured by OCT (Stratus OCT: Carl Zeiss Meditec, Dublin, California), intraocular pressure and other complications at the 1-, 3- and 6-month follow-up visits. Foveal thickness, parafoveal thickness and perifoveal thickness were defined as the mean thicknesses of the central macular region $1000 \mu \mathrm{m}$ in diameter, the paramacular region from $1000 \mu \mathrm{m}$ to $3000 \mu \mathrm{m}$ in diameter, and the perimacular region from $3000 \mu \mathrm{m}$ to $6000 \mu \mathrm{m}$ in diameter, centred on the patient's foveola, respectively, and were obtained by the Retinal Map analysis program with Stratus OCT. In addition, we graded cataract according to the Emery-Little system and defined as "no progression" if the grade was the same during the follow-up period.

Patients were evaluated at baseline and at 1, 3 and 6 months. BCVA, intraocular pressure (IOP), slit-lamp assessment and indirect ophthalmological measurements and OCT were performed at each visit; fluorescein angiography was performed at baseline and 6 months' follow-up.

\section{Sample size}

Based on a pilot study, we estimated the mean change in logMAR BCVA at 6 months' follow-up compared with baseline (primary end-point) to be a worsening of 0.06 (SD 0.18) and an improvement of $0.00(0.13)$ in the two groups. Accordingly, 40 eyes (assuming a few dropouts) in each group were required to achieve a power of $80 \%$ based on an unpaired Student t test with a two-sided significance level of 0.05 .

\section{Statistical analysis}

Analyses were performed on an intention-to-treat basis, unless otherwise indicated. Values are expressed as mean (SD). The significance of the differences between the intervention group and the control group data was assessed by the unpaired Student $t$ test, and that between the pretreatment and posttreatment data within the same group was assessed by the paired Student $t$ test. All statistical analyses were performed using STATA Release 9.02 (Statacorp, Lakeway, Texas). A p value of less than 0.05 was considered to be statistically significant.

\section{RESULTS}

The flow of patients through the study is shown in the supplemental figure (http://bjo.bmj.com/). Forty-five patients were assessed for eligibility, four of whom were not enrolled in the study. Eighty-two eyes of 41 patients with symmetrical

Table 1 Baseline demographics and clinical characteristics

\begin{tabular}{|c|c|c|c|}
\hline Characteristics & Control group & $\begin{array}{l}\text { Posterior sub-Tenon capsule } \\
\text { injection of triamcinolone } \\
\text { acetonide group }\end{array}$ & p Value \\
\hline No of patients (eyes) & \multicolumn{2}{|c|}{41 subjects (each group; 41 eyes) } & - \\
\hline Age (years) & \multicolumn{2}{|l|}{$60.1(11.5)$} & - \\
\hline Female gender, n (\%) & \multicolumn{2}{|l|}{$18(44 \%)$} & - \\
\hline Diabetes type $2, \mathrm{n}(\%)$ & \multicolumn{2}{|l|}{$40(98 \%)$} & - \\
\hline Duration of diabetes (years) & \multicolumn{2}{|l|}{$16.0(5.2)$} & - \\
\hline Haemoglobin A1c (\%) & \multicolumn{2}{|l|}{$8.2(2.1)$} & - \\
\hline Hypertension, n (\%) & \multicolumn{2}{|l|}{$20(49 \%)$} & - \\
\hline Hyperlipidaemia, n (\%) & \multicolumn{2}{|l|}{$21(51 \%)$} & - \\
\hline $\begin{array}{l}\text { Severe non-proliferative diabetic retinopathy/ } \\
\text { proliferative diabetic retinopathy, } \mathrm{n}(\%)\end{array}$ & $29(71 \%) / 12(29 \%)$ & $29(71 \%) / 12(29 \%)$ & - \\
\hline Clinically significant macular oedema, n (\%) & $17(41 \%)$ & $17(41 \%)$ & - \\
\hline Intraocular pressure (mm Hg) & $15.7(3.1)$ & $15.8(3.1)$ & 0.89 \\
\hline LogMAR best-corrected visual acuity & $0.13(0.33)$ & $0.14(0.33)$ & 0.90 \\
\hline Foveal thickness ( $\mu \mathrm{m})$ & $269.0(114.9)$ & $290.2(135.7)$ & 0.45 \\
\hline Parafoveal thickness $(\mu \mathrm{m})$ & $317.6(76.4)$ & $331.7(101.1)$ & 0.49 \\
\hline Perifoveal thickness ( $\mu \mathrm{m})$ & $292.4(59.4)$ & $297.2(78.3)$ & 0.76 \\
\hline
\end{tabular}


severe NPDR or PDR were enrolled. Of the 41 patients, 40 (98\%) completed the study. The demographic and baseline characteristics were comparable between the two groups (table 1).

\section{Visual acuity}

The mean changes in logMAR BCVA at 6 months compared with the baseline (primary end-point) were a worsening of $0.010(0.029)$ in the control group and an improvement of 0.072 $(0.028)$ in the PSTA group $(p=0.04)$ (fig $1 A)$. Figure $1 \mathrm{~B}$ shows the mean logMAR BCVA at each follow-up point among the two groups. In the control group, the mean logMAR BCVA tended to rise from the baseline to 1 month, remain unchanged from 1 month to 3 months and drop from 3 months to 6 months. In contrast, the mean logMAR BCVA in the PSTA group tended to drop from the baseline to 1 month, rise slightly from 1 month to 3 months and drop again from 3 months to 6 months.

\section{Retinal thickness}

The mean changes in foveal thickness at 6 months compared with baseline were an increase of 32.8 (82.8) $\mu \mathrm{m}$ in the control group and a lessening of 9.7 (85.6) $\mu \mathrm{m}$ in the PSTA group $(p=0.03)$. The mean changes in parafoveal thickness at 6 months compared with baseline were a gain of 23.2 (55.0) $\mu \mathrm{m}$ in the control group and a loss of $5.1(66.0) \mu \mathrm{m}$ in the PSTA group $(p=0.04)$. The mean changes in perifoveal thickness at 6 months compared with baseline were a gain of
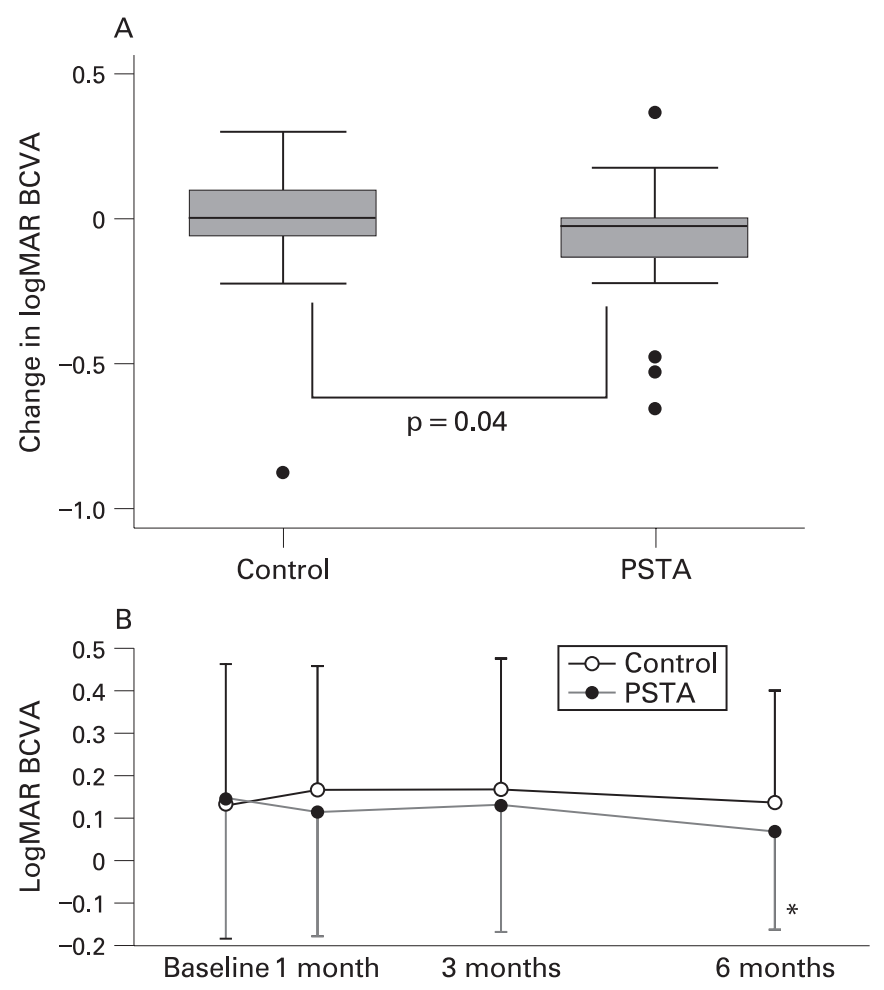

Figure 1 (A) Box plots illustrating the change in logarithm of the minimum angle of resolution (logMAR) visual acuity at 6 months' followup, compared with baseline (primary end-point), of the control group and the posterior sub-Tenon capsule injection of the triamcinolone acetonide (PSTA) group. (B) Line graph comparing the clinical course of logMAR visual acuity between the control group and the PSTA group. Each point and vertical bar indicates mean logMAR visual acuity (SD). * Statistically significant difference $(p<0.05)$ between baseline and each follow-up point within the same group. BCVA, best-corrected visual acuity.
18.3 (44.8) $\mu \mathrm{m}$ in the control group and a gain of 0.5 (34.4) $\mu \mathrm{m}$ in the PSTA group $(p=0.06)$ (fig $2 A$ ). Figure $2 B-D$ shows the mean foveal thickness, parafoveal thickness and perifoveal thickness at each follow-up point among the two groups. In the control group, all subfield (foveal, parafoveal and perifoveal) thicknesses tended to rise from the baseline to 1 month, remain unchanged from 1 month to 3 months and drop from 3 months to 6 months. In contrast, those of the PSTA group tended to drop from the baseline to 1 month, rise slightly from 1 months to 3 months and drop again from 3 months to 6 months.

\section{Intraocular pressure and other complications}

Figure 3 shows the mean intraocular pressure (IOP) throughout the clinical course among the two groups. There were no significant differences in IOP either between baseline and each follow-up point within the same group, or between the two groups at each follow-up point. No other injection-related complications, including cataract progression, were observed.

\section{Subgroup analysis}

We investigated also whether or not the PSTA effect differed by the presence or absence of CSMO. Figure 4 shows the changes in logMAR BCVA and retinal (foveal, parafoveal, perifoveal) thicknesses of the eyes with CSMO and without CSMO in both the control group and the PSTA group. In the eyes without CSMO, the mean changes in logMAR BCVA at 6 months compared with the baseline were a worsening of $0.020(0.12)$ in the control group and an improvement of 0.04 (0.13) in the PSTA group, and the mean changes in foveal thickness at 6 months compared with the baseline were an increase of 34.9 (56.5) $\mu \mathrm{m}$ in the control group and an increase of 9.7 (85.6) $\mu \mathrm{m}$ in the PSTA group. In the eyes with CSMO, the mean changes in $\log M A R$ BCVA at 6 months compared with the baseline were a worsening of $0.00(0.25)$ in the control group and an improvement of $0.12(0.22)$ in the PSTA group, and the mean changes in foveal thickness at 6 months compared with the baseline were an increase of 29.5 (114.4) $\mu \mathrm{m}$ in the control group and a lessening of 42.7 (132.4) $\mu \mathrm{m}$ in the PSTA group. Retinal (foveal, parafoveal or perifoveal) thickness of the eyes with CSMO in the PSTA group remarkably decreased from the baseline to 1 month. In summary, visual acuity and retinal thickness in the PSTA group tended to be superior to those in the control group, without regard to CSMO.

\section{DISCUSSION}

It is well known that PRP can decrease the incidence of visual loss or blindness by preventing subsequent neovascularisation in eyes of patients with severe NPDR and PDR. However, PRP occasionally results in a transient or even a sustained reduction in visual function, which is often caused by a worsening macular oedema. In cases with CSMO before PRP, close attention needs to be paid because CSMO often occurs in those eyes in which PRP is felt to be necessary. There are many reports that triamcinolone acetonide is effective against diabetic macular oedema when administered by intravitreal or subTenon capsule injection, even though its effect may be transitory. ${ }^{17-21}$ To test the advantages of sub-Tenon capsule injection of triamcinolone prior to PRP in patients with severe NPDR or PDR, we designed this randomised controlled trial using paired eyes of patients with bilateral severe NPDR or PDR in order to reduce any possible bias due to various systemic factors. 
Figure 2 (A) Box plots illustrating the change in foveal thickness, parafoveal thickness, and perifoveal thickness at 6 months' follow-up, compared with baselines, in the control group and in the posterior sub-Tenon capsule injection of triamcinolone acetonide (PSTA) group. Line graph comparing the clinical course of foveal thickness (B), parafoveal thickness (C), and perifoveal thickness (D) between the control group and the PSTA group. Each point and vertical bar indicates the mean (SD). *Statistically significant difference $(p<0.05)$ between baseline and each follow-up point within the same group.
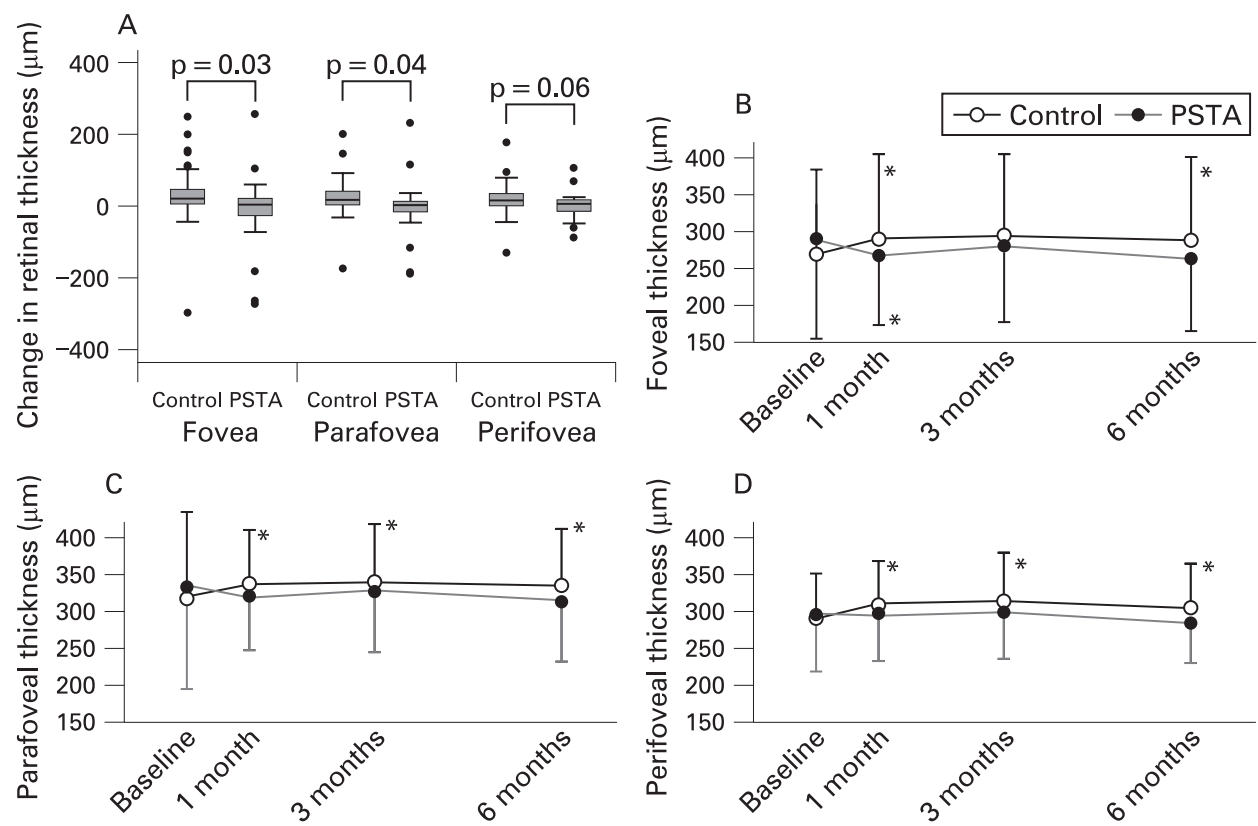

In the present study, the change in logMAR BCVA at 6 months compared with that at baseline (primary end-point) in the PSTA group was statistically significantly less than that in the control group. logMAR BCVA in the control group tended to rise from the baseline to 1 month, remain unchanged from 1 month to 3 months and drop from 3 months to 6 months, while that in the PSTA group tended to drop from the baseline to 1 month, rise slightly from 1 month to 3 months and drop from 3 months to 6 months. In addition, the change in foveal thickness at 6 months compared with the baseline measurement in the PSTA group was statistically significantly less than that in the control group. Considering these results together, it is possible that PSTA prevents PRP-induced macular thickening, and thereby results in less visual loss. Moreover, $\log$ MAR BCVA and retinal (foveal, parafoveal or perifoveal) thickness tended to drop from 3 months to 6 months in both groups, although this may be the natural course after the completion of PRP. The progression of logMAR BCVA and retinal thickness of the two groups differed widely until the 3month examination. Therefore, we deduced that an advantage of PSTA is the control of visual decline and retinal thickening until a few weeks after PRP completion.

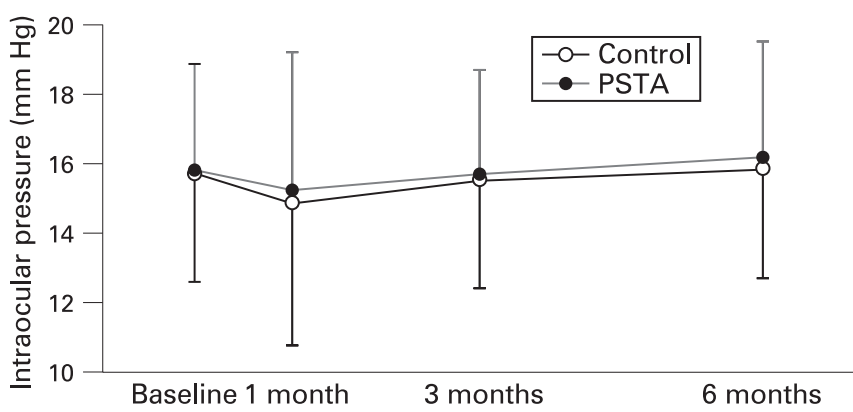

Figure 3 Line graph comparing the clinical course of intraocular pressure (IOP) between the control group and the posterior sub-Tenon capsule injection of triamcinolone acetonide (PSTA) group. No significant difference was seen between the control group and the PSTA group at any follow-up point.
We also investigate whether or not the PSTA effect was affected by the presence of CSMO. Regardless of the presence or absence of CSMO, changes in logMAR BCVA and retinal thickness in the PSTA group tended to be less than those in the control group. However, we could not conclude anything from this because the sample size was insufficient for subgroup analysis.

The focal/grid laser was performed in all eyes with CSMO without regard to the assignment. The number of the eyes with CSMO was the same in the two groups, because we did not include the patients with CSMO in only one eye. However, it was hardly possible to perform focal/grid laser in a standardised condition for each individual eye. This may be one of the factors that cause bias in the results between the two groups.

Adverse events that may accompany periocular injection of triamcinolone acetonide include increases in IOP and cataract formation. ${ }^{22-24}$ In the present study, the mean IOP of eyes in the PSTA group was neither statistically significantly different between baseline and each follow-up visit nor statistically significantly different from that of eyes in the control group at each follow-up visit. This supports the fact that increased IOP is reported often following intravitreal administration of TA, although it is rarely reported following sub-Tenon administration of TA. ${ }^{23}{ }^{24}$ In addition, no eye had cataract progression during our study period. However, we cannot conclude that a single sub-Tenon capsule injection of triamcinolone acetonide had no influence on cataract formation because steroid-induced cataract formation may take longer than 6 months to become apparent. Other potential complications of PSTA, such as blepharoptosis, orbital fat atrophy, strabismus and conjunctival necrosis, have been reported. ${ }^{23-25}$ However, none of these complications were observed during the study period.

One of the limitations of the study described herein is the use of an observation arm as control, rather than a sham injection, thereby making it impossible to ensure that the patient and investigators were masked with regard to treatment. However, this limitation was mitigated by ensuring that the technicians who performed the visual-acuity assessment and OCT were masked. Other limitations of this study are that it was 
Figure 4 Box plots illustrating the change in logarithm of the minimum angle of resolution ( $\log M A R)$ best-corrected visual acuity (BCVA) (A) and foveal thickness (B) at 6 months compared with baseline in eyes with clinically significant macular oedema (CSMO) and without CSMO in the control group and in the posterior sub-Tenon capsule injection of triamcinolone acetonide (PSTA) group. Line graph comparing the clinical course of logMAR BCVA (C) and foveal (D), parafoveal (E), perifoveal $(F)$ thicknesses of eyes with CSMO and without CSMO, between the control group and the PSTA group.
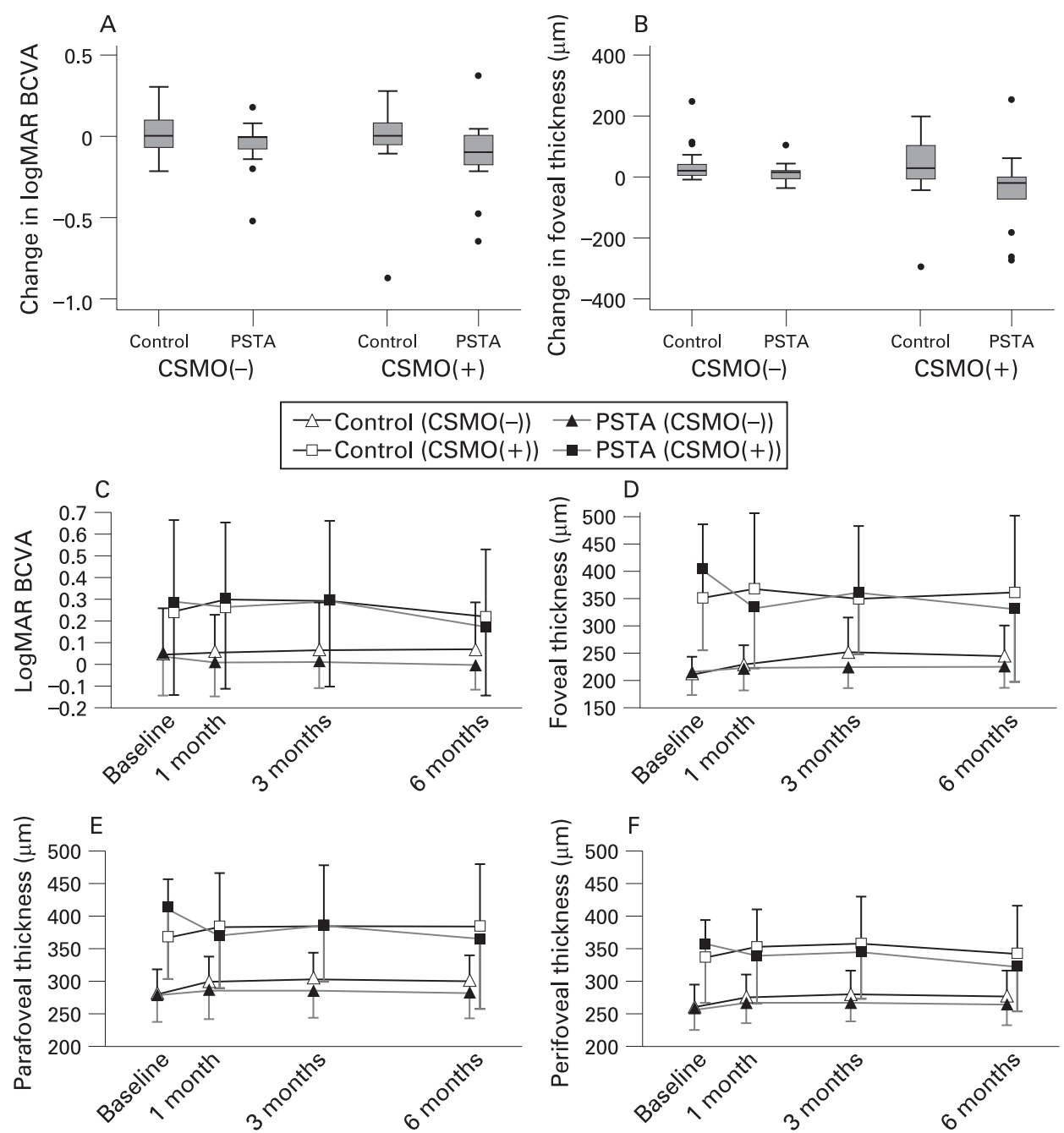

performed at a single centre, and that it involved individuals of only one race, factors that limit its generalizability.

In summary, our study found evidence of a benefit of PSTA as a pre-PRP treatment for the eyes with diabetic retinopathy in which PRP was deemed necessary. PSTA may thus be considered an optional treatment to control PRP-induced visual decrease and macular thickening. However, the difference of the change in logMAR BCVA at 6 months compared with that at baseline between the PSTA group and the control group was small in our findings. The actual clinical relevance remains to be determined in future larger trials.

Funding: Supported by a Grant-in-Aid for Scientific Research from the Ministry of Education, Science and Culture of the Japanese government.

Competing interests: None.

Ethics approval: Ethics approval was provided by the Ethics Committee at Kyoto University.

Patient consent: Obtained.

\section{REFERENCES}

1. Early Treatment Diabetic Retinopathy Study Research Group. Early photocoagulation for diabetic retinopathy, ETDRS report number 9. Ophthalmology 1991;98:766-85

2. Diabetic Retinopathy Study Research Group. Preliminary report on effects of photocoagulation therapy. Am J Ophthalmol 1976;81:383-96.

3. Diabetic Retinopathy Study Research Group. Photocoagulation treatment of proliferative diabetic retinopathy. Clinical application of Diabetic Retinopathy Study (DRS): DRS report 8. Ophthalmology 1981;88:583-600.
4. McDonald HR, Schatz H. Macular edema following panretinal photocoagulation. Retina 1985;5:5-10

5. McDonald HR, Schatz H. Visual loss following panretinal photocoagulation for proliferative diabetic retinopathy. Ophthalmology 1985;92:388-93.

6. Ferris FL 3rd, Podgor MJ, Davis MD. Macular edema in Diabetic Retinopathy Study patients. Diabetic Retinopathy Study report number 12. Ophthalmology 1987:94:754-60.

7. Shimura M, Yasuda K, Nakazawa T, et al. Quantifying alterations of macular thickness before and after panretinal photocoagulation in patients with severe diabetic retinopathy and good vision. Ophthalmology 2003;110:2386-94.

8. Nonaka A, Kiryu J, Tsujikawa A, et al. Inflammatory response after scatter laser photocoagulation in nonphotocoagulated retina. Invest Ophthalmol Vis Sci 2002;43:1204-9.

9. Tsujikawa A, Kiryu J, Dong J, et al. Quantitative analysis of diabetic macular edema after scatter laser photocoagulation with the scanning retinal thickness analyzer. Retina 1999;19:59-64.

10. Del Maschio A, Zanetti A, Corada M, et al. Polymorphonuclear leukocyte adhesion triggers the disorganization of endothelial cell-to-cell adherens junctions. J Cell Biol 1996;135:497-510.

11. Tamura H, Miyamoto K, Kiryu J, et al. Intravitreal injection of corticosteroid attenuates leukostasis and vascular leakage in experimental diabetic retina. Invest Ophthalmol Vis Sci 2005;46:1440-4.

12. Funatsu $\mathbf{H}$, Yamashita $H$, Ikeda $T$, et al. Relation of diabetic macular edema to cytokines and posterior vitreous detachment. Am J Ophthalmol 2003:135:321-7.

13. Wilson CA, Berkowitz BA, Sato Y, et al. Treatment with intravitreal steroid reduces blood-retinal barrier breakdown due to retinal photocoagulation. Arch Ophthalmol 1992;110:1155-9.

14. Shimura M, Yasuda K, Shiono T. Posterior sub-Tenon's capsule injection of triamcinolone acetonide prevents panretinal photocoagulation-induced visual dysfunction in patients with severe diabetic retinopathy and good vision. Ophthalmology 2006:113:381-7.

15. Bandello F, Polito A, Pognuz DR, et al. Triamcinolone as adjunctive treatment to laser panretinal photocoagulation for proliferative diabetic retinopathy. Arch Ophthalmol 2006;124:643-50. 
16. Zacks DN, Johnson MW. Combined intravitreal injection of triamcinolone acetonide and panretinal photocoagulation for concomitant diabetic macular edema and proliferative diabetic retinopathy. Retina 2005;25:135-40.

17. Bonini-Filho MA, Jorge R, Barbosa JC, et al. Intravitreal injection versus sub-Tenon's infusion of triamcinolone acetonide for refractory diabetic macular edema: a randomized clinical trial. Invest Ophthalmol Vis Sci 2005; 46:3845-9.

18. Cardillo JA, Melo LA Jr, Costa RA, et al. Comparison of intravitreal versus posterior sub-Tenon's capsule injection of triamcinolone acetonide for diffuse diabetic macular edema. Ophthalmology 2005;112:1557-63.

19. Sutter FK, Simpson JM, Gilles MC. Intravitreal triamcinolone for diabetic macular edema that persists after laser treatment: three-month efficacy and safety results of a prospective, randomized, double-masked, placebo-controlled clinical trial. Ophthalmology 2004;111:2044-9.
20. Martidis A, Duker JS, Greenberg PB, et al. Intravitreal triamcinolone for refractory diabetic macular edema. Ophthalmology 2002;109:920-7.

21. Verma LK, Vivek MB, Kumar A, et al. A prospective controlled trial to evaluate the adjunctive role of posterior subtenon triamcinolone in the treatment of diffuse diabetic macular edema. J Ocul Pharmacol Ther 2004;20:277-84.

22. Butcher JM, Austin M, McGalliard J, et al. Bilateral cataracts and glaucoma induced by long term use of steroid eye drops. BMJ 1994;309:43.

23. Mueller AJ, Jian G, Banker AS, et al. The effect of deep posterior subtenon injection of corticosteroids on intraocular pressure. Am J Ophthalmol 1998;125:158-63.

24. Iwao K, Inatani M, Kawaji T, et al. Frequency and risk factors for intraocular pressure elevation after posterior sub-Tenon capsule triamcinolone acetonide injection. J Glaucoma 2007:16:251-6.

25. Agrawal S, Agrawal J, Agrawal TP. Conjunctival ulceration following triamcinolone injection. Am J Ophthalmol 2003;136:539-40. 